\title{
Challenges in diagnosing odontogenic lesions in maxillary sinuses
}

Authors' Contribution: A-Study Design B-Data Collection C-Statistical Analysis D-Data Interpretation E-Manuscript Preparation E-Manuscript Preparatio -Literature Search

\author{
Katarzyna Dobroś ${ }^{A B C D E F}$, Joanna Zarzecka ${ }^{A D}$ \\ Department of Conservative Dentistry with Endodontics, Institute of Dentistry, Faculty of Medicine, Jagiellonian University Medical \\ College; Head: Joanna Zarzecka, DDM, PhD, professor
}

Article history: Received: 30.09.2019 Accepted: 06.11.2019 Published: 14.11.2019

ABSTRACT:

Objectives: The study aimed to identify the patients with pathological lesions in the maxillary sinuses in which the reported symptoms might be indicative of odontogenic origin, as well as to establish specific causative risk factors promoting their development.

Methods and Materials: The study covered 44 patients with suspected odontogenic maxillary sinusitis. Dental examination and Cone Beam Computed Tomography were completed. The age of patients ranged between 19 and 69 years, and the mean age was $43(S D=13.9)$ years.

Results: Out of 44 patients, 22 (50\%) had non-odontogenic lesions in maxillary sinuses, while in 15 (34.1\%) dental origin was established. In the remaining 7 (15.9\%) patients, no pathological changes were found in the sinuses. The median of reported symptoms was 10 months $\left(Q_{1}=4, Q_{3}=24\right)$. The reported complaints were not associated with the actual cause of pathological lesions.

Conclusions: Odontogenic cause of the lesions in the sinuses should primarily be hypothesized, especially in patients with long-term disease symptoms, also regarding any unilateral inflammations.

KEYWORDS: $\quad$ Cone Beam Computed Tomography, maxillary sinusitis, periapical tissues, pulp disease

\section{ABBREVIATION}

CBCT - Cone Beam Computed Tomography

CT - Computed Tomography

\section{INTRODUCTION}

Odontogenic maxillary sinusitis makes a rather diverse group. It may be caused by surgical treatment complications, as well as by pulp disease, inflammatory lesions in periapical tissues, periodontium disease, and complications of endodontic treatment [1-5]. In line with the findings of the review made by Patel et al., there are no current references accurately pinpointing the actual percentage of odontogenic factors in the development of chronic sinusitis [6]. Various authors estimate it to be in the range of $10-40 \%[1,6,7]$.

The close proximity of teeth and maxillary sinuses is believed to facilitate the spreading of odontogenic infections through the bone of the alveolar ridge, or directly in result of a discontinuity in the sinus floor [8]. Complications following surgical treatment remain the most common causative factor in the development of odontogenic maxillary sinusitis $[2,9,10]$. Complications in the area of maxillary sinuses resultant from pulp disease, and inflammation of periapical tissues, make a separate group. Inflammatory lesions in the periapical tissues are detected through radio- logical examination, whereas pulp diseases may be detected with the aid of clinical examination only. Pulp diseases are caused by a bacterial infection, by far the most common complication of deep caries, although even in the teeth cured of caries, diseases of the pulp and periapical tissues may well develop as late complications.

In a survey made among otorhinolaryngologists, Longhini et al. pointed out that odontogenic causes of sinusitis were quite frequent, even if initially eluding diagnosis. The authors emphasized that radiologists very seldom, if ever, described tooth pathology [11]. This may also be due to the fact that the Computed Tomography (CT) imaging technique, as applied in diagnosing sinusitis, is not sufficiently precise to assess the teeth. Nowadays, new diagnostic possibilities have emerged thanks to a widespread availability of Cone Beam Computed Tomography (CBCT) [12], which facilitates the accurate assessment of maxillary sinuses, teeth and periapical tissues, and therefore makes it possible to have the pathological lesions in the sinuses linked with the ones in periapical tissues $[13,14]$.

The present study aimed to identify the patients with pathological lesions in the maxillary sinuses in whom the reported symptoms might be indicative of odontogenic origin, as well as to establish specific causative risk factors promoting their development. An attempt was also made at having the established pathology effectively linked with the earlier scope of treatment. 


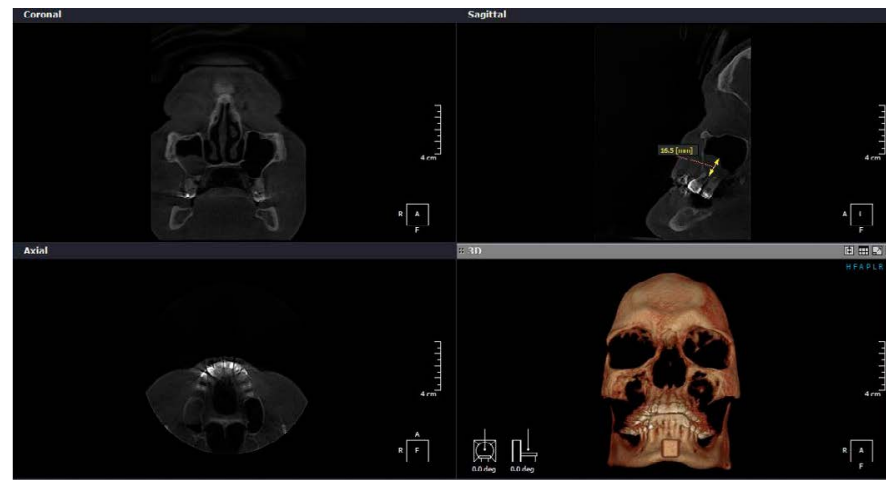

Fig. 1a. Measurement of the thickening of the mucous membrane in the sagittal plane.

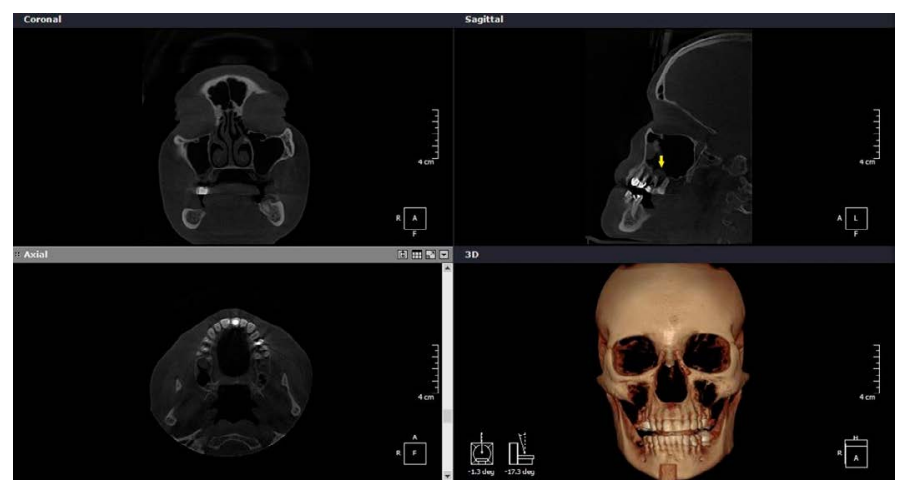

Fig. 2. The first upper left molar with no inflammation in periapical tissues, visible thickening of the mucous membrane of the sinus floor cavity in the projection of the root apex.

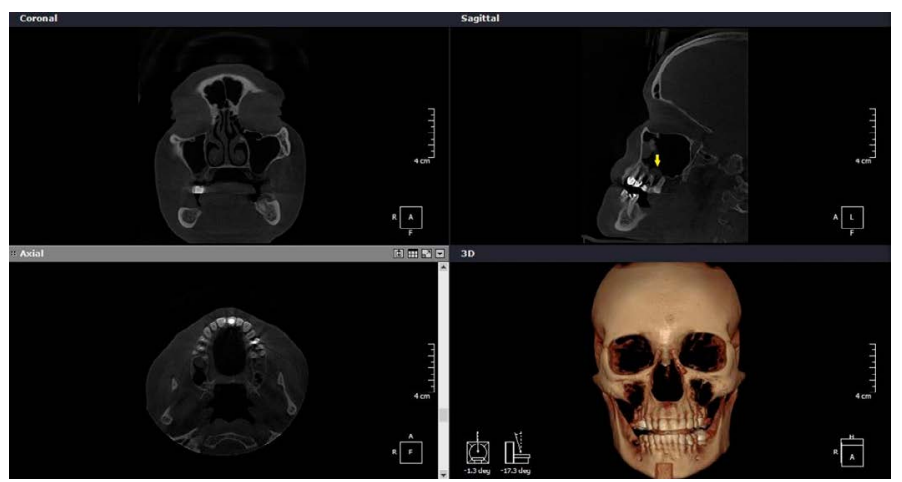

Fig. 4. The first upper left molar and second molar after incomplete root canal treatment, visible thickening of the mucous membrane of the sinus floor cavity in the projection of the root apex.

\section{METHODS AND MATERIALS}

Laryngologists associated in the Regional Branch of the Otolaryngology Society were requested to refer patients with suspected odontogenic maxillary sinusitis to the Dept. of Conservative Dentistry with Endodontics. The following exclusion criteria were applied: persons with oro-antral communication or fistula as a result of improper surgical treatment; additionally: toothlessness, age under 18 years, pregnancy, general diseases, i.e. confirmed allergy (aspirin asthma), massive nasal polyps, neoplastic diseases, autoimmune diseases. In the period spanning June 2014 - December 2016, 44 patients (29 women, 15 men) reported to the Department. Their age ranged between 19 and 69 years, and the mean age was $43(\mathrm{SD}=13.9)$ years. All patients were advised on

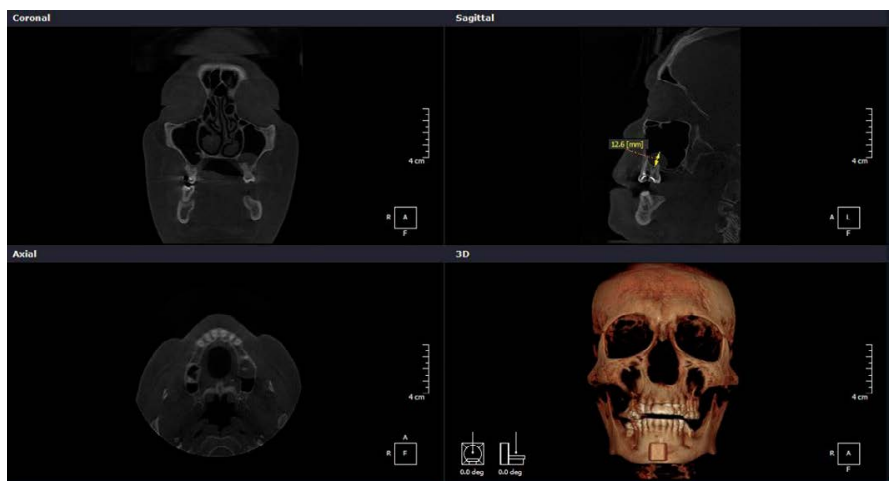

Fig. 1b. Measurement of the cyst diameter in the sagittal plane.

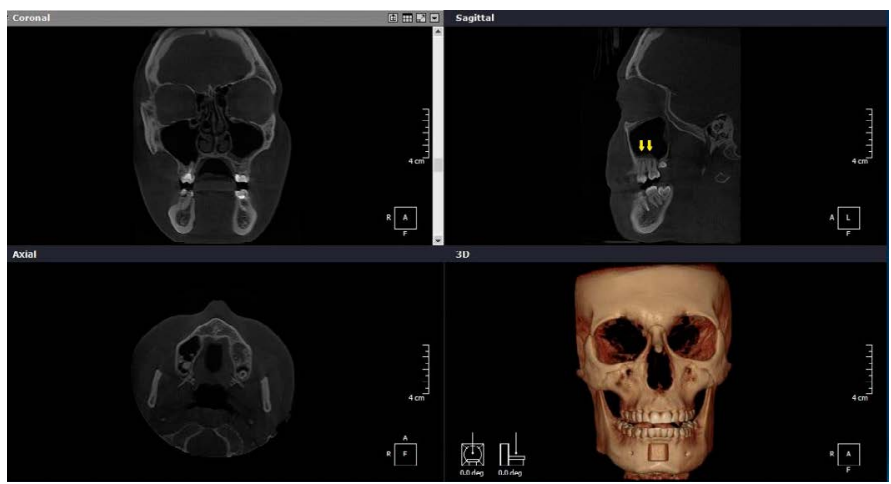

Fig. 3. The first upper left molar and second molar with an inflammation in periapical tissues, visible thickening of the mucous membrane of the sinus floor cavity in the projection of the root apex.

the study protocol accordingly, and produced written, informed consent to participate.

The description of patients' complaints and their duration was acquired through an interview. CBCT scans were taken using a volumetric imaging system (RayScan Symphony Ray Co., Ltd., Korea). All images were assessed by a single observer (a specialist in conservative dentistry with endodontics, with 4 years of experience in CBCT diagnostics), under standardized conditions, at the same examination workplace using Xelis Dental - CD Viewer - Dental 3D - Pro. The lesions in maxillary sinuses, based on radiographic imaging, were categorized either as no lesions, a thickening of the mucous membrane, or a cyst. Both the diameter of a cyst, and the thickening of the mucous membrane, were measured at their widest point (Fig. 1a., 1b.).

Following a clinical examination, assessment of the teeth's vitality with a thermal test (reaction to ethyl chloride), and comprehensive evaluation of CBCT images, an odontogenic origin of the lesions in the maxillary sinuses was either confirmed or ruled out. Teeth with any pulp diseases (Fig. 2.), inflammatory lesions in periapical tissues (Fig. 3.), or the ones revealing incomplete root canal treatment (i.e. missed out, or unfilled root canals) were deemed the causative teeth (Fig. 4.).

All categorical variables were reported as numbers and percentages. Continuous variables were presented as means (standard deviation), or median (interquartile range), as appropriate. The Shapiro-Wilk test was carried out with normal distribution. The 


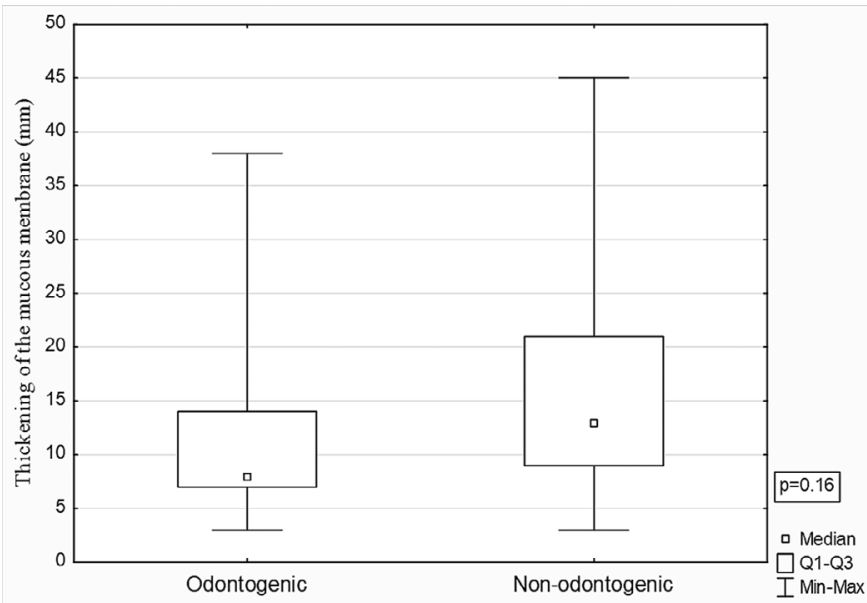

Fig. 5a. Thickening of the mucous membrane-odontogenic and non-odontogenic lesions

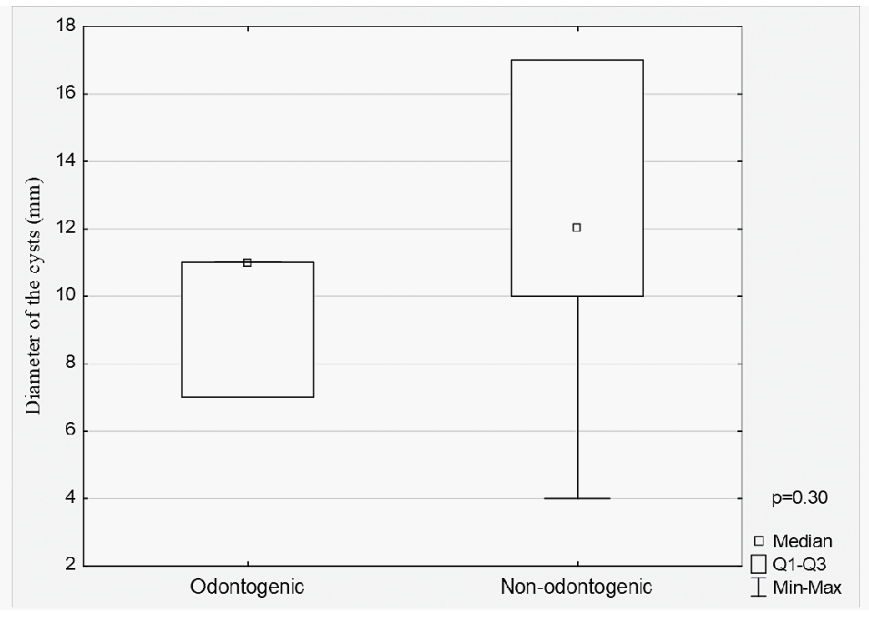

Fig. 5b. Diameter of the cysts - odontogenic and non-odontogenic lesions.

Tab. I. The incidence of pathological lesions in maxillary sinuses and the presence of inflammation in periapical tissues.

\begin{tabular}{|c|c|c|c|c|c|c|c|c|c|}
\hline & & \multicolumn{3}{|c|}{ RIGHT SINUS } & \multicolumn{5}{|c|}{ LEFT SINUS } \\
\hline & & No lesions & $\begin{array}{l}\text { Mucous membrane } \\
\text { thickening }\end{array}$ & Cyst & $\mathrm{p}$ & No lesions & $\begin{array}{l}\text { Mucous membrane } \\
\text { thickening }\end{array}$ & Cyst & $\mathrm{p}$ \\
\hline \multirow{2}{*}{ Tooth } & No periapical lesions & $20(90.9 \%)$ & $10(58.8 \%)$ & $5(100 \%)$ & \multirow{2}{*}{$0.02 *$} & $15(93.8 \%)$ & $15(65.2 \%)$ & $2(40 \%)$ & \multirow{2}{*}{$0.02^{*}$} \\
\hline & Periapical lesions & $2(9.1 \%)$ & $7(41.2 \%)$ & $\mathrm{O}(0.0 \%)$ & & $1(6.3 \%)$ & $8(34.8 \%)$ & $3(60 \%)$ & \\
\hline
\end{tabular}

"p $<0,05$

Chi-square test was used to compare categorical variables. The differences in distribution of "mucous thickening" between "odontogenic, and non-odontogenic" were compared by Mann Whitney $\mathrm{U}$ test. All data analyses were done using Statistica v. 12 software. $\mathrm{P}$-values $<0.05$ were accepted as statistically significant.

The study protocol was approved by the University Bioethics Review Committee (Ref. No. KBET/34/B/204 and No. 122.6120.141.2015). Imaging diagnostics were financed out of the statutory project's resources (Ref. no. K/ZDS/004589).

\section{RESULTS}

Based on a clinical study and an assessment of CBCT images, $22(50 \%)$ patients, out of 44 , were diagnosed with non-odontogenic lesions, 12 - unilateral, and 10 - located on both sides. In the case of non-odontogenic lesions, a thickening of the mucous membrane was noted in $15(68.2 \%)$ patients, cysts in $4(18.2 \%)$, whereas in $3(13.6 \%)$ cases of bilateral inflammations, both changes were encountered.

Nevertheless, in 15 (34.1\%) patients odontogenic lesions were diagnosed in the sinuses, in 14 cases - unilaterally, and in a single case - on both sides. Further assessment of the patients with odontogenic lesions revealed $12(80 \%)$ with a thickening of the mucous membrane of the sinus floor, and 3 (20\%) with cysts. In the remaining 7 (15.9\%) patients no pathological lesions were noted. In the radiological image, the non-odontogenic lesions proved larger than the odontogenic ones (Fig. 5a., 5b.).

The symptoms reported by patients were diverse. Patients usually complained of headache (50\%), a flowing secretion from the back of the throat (43.2\%), facial pain (43.2\%), and nasal obstruction (40.9\%). The median duration of the reported complaints was 10 months $(\mathrm{Q} 1=4, \mathrm{Q} 3=24)$. Patients with odonotogenic lesions more often complained of nasal obstruction, facial pain, and a flowing secretion from the back of the throat; even though this difference was statistically insignificant ( $\mathrm{p}>0.05)$ (Fig. 6.).

In 15 patients, an odontogenic origin of the pathological lesions in maxillary sinus was confirmed, 11 of them had more than one causative tooth. All 15 patients had 33 causative teeth. Eight patients had teeth with an inflammation of periapical tissues, including teeth after incomplete endodontic treatment. Noteworthy are another 2 patients in whom no inflammatory reaction was observed in periapical tissues, while the causal teeth revealed incomplete root canal treatment. The remaining 5 patients had teeth with pulp diseases without any inflammation in periapical tissues, but in 3 cases those teeth co-occurred with the teeth with changes in periapical tissues. It was established that inflammatory changes in periapical tissues were associated with a more frequent development of lesions in the maxillary sinuses $(\mathrm{p}<0.05)$ (Tab. I.).

Out of the 15 patients with lesions of dental origin, in 14 the causal teeth were referred for endodontic treatment, and in 1 patient - for extraction. Only 1 patient required surgery after previous endodontic treatment of causative teeth. In the remaining patients, overall improvement and disappearance of the above-referenced symptoms was secured, following a root canal treatment, or an extraction of causal teeth. Patients with the non-odontogenic lesions and with no changes in the maxillary sinuses, were referred to an otolaryngologist. 


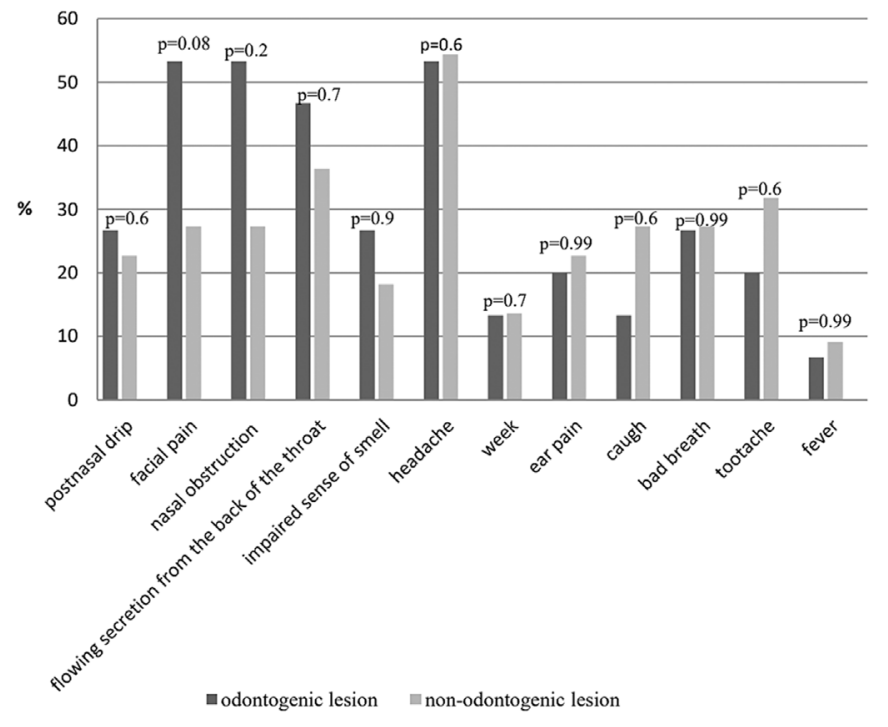

Fig. 6. Frequency of symptoms, cause of lesions.

\section{DISCUSSION}

The symptoms reported by patients were characteristic of chronic maxillary sinusitis. Their persistent nature at the time of referral to the dentist, seemed clearly indicative of the odontogenic causes not having been given any consideration whatsoever in the initial phase of the treatment management. Hoskison et al. emphasized that over the years the number of patients with odontogenic sinusitis increased [15]. In a survey conducted among otolaryngologists, Longhini et al. pointed out that odontogenic causes of sinusitis were frequent, although initially misdiagnosed [11]. In the present study, the average persistence of symptoms was around 10 months. Regretfully enough, no association was established within the study group between the reported ailments and the actual etiology of the sinus lesions.

The presence of fluid in the sinus, endodontic material in periapical tissues, or surgical treatment complications pose no problems in CT diagnostics. However, comprehensive imaging of any pathological changes in the teeth and the sinus floor may effectively be facilitated by CBCT only. Furthermore, even the absence of changes in periapical tissues may not rule out their odontogenic origin. Pulp diseases causing sinusitis may effectively be diagnosed with the aid of clinical examination only, whereupon a vitality test is crucial. In the present study, 11 patients had more than one causal tooth, and the most common cause of odontogenic lesions was periapical tissue inflammation. In 2 patient's sinusitis developed as a result of pulp diseases only, whereas in the case of 3 patients, the teeth with pulp diseases co-occurred with the ones with changes in periapical tissues.

Brullmann et al. pursued a retrospective analysis of the medical records and $\mathrm{CBCT}$ in 204 patients, in which they demonstrated that any decayed and non-vital teeth contributed to the changes in the mucous membrane of the sinus floor [13]. Matsumoto et al. carried out a retrospective evaluation of medical records, $\mathrm{CT}$ and OPG in 190 patients with unilateral sinusitis, whereupon they established that in $80 \%$ of cases, the inflammation was of odontogenic origin, while in assessing the potentially odontogenic cause they took into account the result of the tooth vitality test [16]. In our own study, odontogenic sinusitis was bilateral in a single case only.

The substandard quality of endodontic treatment should also be considered a potential risk factor in the development of odontogenic inflammatory changes in the sinuses. This happened to be the case in our own study, where incomplete root canal treatment was encountered in 9 patients with causative teeth. Not in all cases, however, the inflammatory lesions in the periapical tissues were found. It should be highlighted, though, that inadequately filled-in root canals, or the ones omitted altogether, may easily become a potential source of an infection. Due to a specific structure of the maxillary bones, bacteria encountered in the untreated root canals may cause infections and spread across the bones, while finding easy access through the Havers and Volkmann channels. As nowadays the number of patients opting for endodontic treatment is on the rise, it may well be expected that the number of complications in the form of inflammations of the maxillary sinus would follow closely.

Persistent symptoms of sinusitis should prompt the attending dental surgeons to order a much wider scope of diagnostics, and incorporate dental examination and $\mathrm{CBCT}$ as the standard procedures.

\section{CONCLUSIONS}

Inflammation of periapical tissues, pulp diseases, and incomplete root canal treatment are instrumental in the development of odontogenic lesions in the maxillary sinuses. Odontogenic origin should therefore be given primary consideration, while assessing pathological changes in the maxillary sinus, particularly in patients with a chronic character of the disease, as well as in the cases of a unilateral inflammation. A thorough dental examination, in conjunction with CBCT imaging, is believed to facilitate effective detection of such lesions.

\section{REFERENCES}

1. Mehra P., Jeong D.: Maxillary Sinusitis of Odontogenic Origin. Curr Allergy Asthma Rep., 2009; 9: 238-243.

2. Troeltzsch M., Pache C., Troeltzsch M., Kaeppler G., Ehrenfeld M. et al.: Etiology and clinical characteristics of symptomatic unilateral maxillary sinusitis: A review of 174 cases. J Craniomaxillofac Surg., 2015; 43: 1522-1529.

3. Feng L., Li H., Ling-Ling E., Li C.J., Yan D.: Pathological changes in the maxillary sinus mucosae of patients with recurrent odontogenic maxillary sinusitis. Pakistan J Med Sci., 2014; 30(5): 972-975.

4. Hauman C.H., Chandler N.P., Tong D.C.: Endodontic implications of the maxillary sinus: a review. Int Endod J., 2002; 35(2): 127-141.

5. Nimigean V.R., Nimigean V., Maru N., Andressakis D., Balatsouras D.G. et al: The maxillary sinus and its endodontic implications: clinical study and review. B-ENT., 2006; 2(4): 167-175.

6. atel N.A., Ferguson B.J.: Odontogenic sinusitis: an ancient but under-appreciated cause of maxillary sinusitis. Curr Opin Otolaryngol Head Neck Surg., 2012; 20(1): 24-28. 
7. Simuntis R., Kubilius R., Vaitkus S.: Odontogenic maxillary sinusitis: a review. Stomatologija, Baltic Dental and Maxillofacial Journal., 2014; 16(2): 39-43.

8. Uliasz M., Wanyura H.: Pathway of odontogenous maxillary sinusitis expansion to other paranasal sinuses. J Stoma., 2009; 62(11): 892-904.

9. Akhlaghi F., Esmaeelinejad M., Safai P.: Etiologies and Treatments of Odontogenic Maxillary Sinusitis: A Systematic Review. Iran Red Crescent Med J., 2015; 17(12): e25536.

10. Arias-Irimia O., Barona-Dorado C., Santos-Marino J.A., Martinez-Rodriguez N., Martinez-Gonzalez J.M.: Meta-analisis of the etiology of odontogenic maxillary sinusitis. Med Oral Patol Oral Cir Bucal., 2010; 15(1): 3-6.

11. Longhini A.B., Branstetter B.F., Ferguson B.J.: Otolaryngologists' perceptions of odontogenic maxillary sinusitis. Laryngoscope., 2012; 122(9): 1910-1914.

12. Mozzo P., Procacci C., Tacconi A., Martini P.T., Andreis I.A.B.: A new volumetric CT machine for dental imaging based on the cone-beam technique: preliminary results. Eur Radiol., 1998; 8(9): 1558-1564.
13. Brüllmann D.D., Schmidtmann I., Hornstein S., Schulze R.K.: Correlation of cone beam computed tomography (CBCT) findings in the maxillary sinus with dental diagnoses: A retrospective cross-sectional study. Clin Oral Investig., 2012; 16(4): 1023-1029.

14. Maillet M., Bowles W.R., McClanahan S.L., John M.T., Ahmad M.: Cone-beam computed tomography evaluation of maxillary sinusitis. J Endod., 2011; 37(6): 753-757.

15. Hoskison E., Daniel M., Rowson J.E., Jones N.S.: Evidence of an increase in the incidence of odontogenic sinusitis over the last decade in the UK. J Laryngol Otol., 2012; 126(1): 43-46.

16. Matsumoto Y., Ikeda T., Yokoi H., Kohno N.: Association between odontogenic infections and unilateral sinus opacification. Auris Nasus Larynx., 2015; 42(4): $288-293$

Word count: $2510 \quad$ Tables: $1 \quad$ Figures: $6 \quad$ References: 16

DOI: $\quad$ 10.5604/01.3001.0013.5566 Table of content: https://otolaryngologypl.com/issue/13074

Copyright: Copyright @ 2020 Polish Society of Otorhinolaryngologists Head and Neck Surgeons. Published by Index Copernicus Sp. z o.o. All rights reserved

Competing interests: The authors declare that they have no competing interests.

2 The content of the journal „Polish Society of Otorhinolaryngologists Head and Neck Surgeons” is circulated on the basis of - the Open Access which means free and limitless access to scientific data.
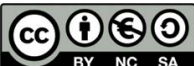

Corresponding author:

Cite this article as:
This material is available under the Creative Commons - Attribution 4.0 GB. The full terms of this license are available on: http://creativecommons.org/licenses/by-nc-sa/4.0/legalcode

Katarzyna Dobroś, DDS; Department of Conservative Dentistry with Endodontics, Institute of Dentistry, Faculty of Medicine, Jagiellonian University Medical College; Montelupich St. 4, 31-155 Krakow, Poland; Phone: +48 12-424-54-25; E-mail: katarzyna.dobros@uj.edu.pl

Dobros K., Zarzecka J.: Challenges in diagnosing odontogenic lesions in maxillary sinuses;

Otolaryngol Pol 2020; 74 (3): 12-16 